Published in final edited form as:

Trends Mol Med. 2019 April ; 25(4): 328-340. doi:10.1016/j.molmed.2019.01.002.

\title{
MACROPHAGES IN HEART FAILURE WITH REDUCED VERSUS PRESERVED EJECTION FRACTION
}

\author{
Matthew DeBerge ${ }^{1,2, *}$, Sanjiv J. Shah ${ }^{2,3}$, Lisa Wilsbacher ${ }^{2,3}$, and Edward Thorp ${ }^{1,2}$ \\ ${ }^{1}$ Department of Pathology, The Feinberg School of Medicine, Northwestern University \\ ${ }^{2}$ Feinberg Cardiovascular and Renal Research Institute, The Feinberg School of Medicine, \\ Northwestern University \\ ${ }^{3}$ Department of Medicine, The Feinberg School of Medicine, Northwestern University
}

\begin{abstract}
There is a growing number of individuals living with heart failure (HF) with reduced ejection fraction (HFrEF) or preserved ejection fraction (HFpEF). Long-term prognosis remains poor in both cases-especially in HFpEF, which is rising in incidence and lacks effective therapeutics. In both HFrEF and HFpEF, there is evidence that elevated inflammatory biomarkers, implicating innate immune cells such as macrophages, are associated with worsened clinical outcomes. Macrophage subsets are active in both inflammatory and reparative processes, yet our understanding of the causative roles for these cells in HF development and progression is incomplete. Here we discuss recent findings interrogating the role of macrophages in inflammation and its resolution in the context of HF, with a specific focus on HFrEF versus HFpEF.
\end{abstract}

\section{Keywords}

Macrophage; Inflammation; Heart Failure; HFrEF; HFpEF

\section{INFLAMMATORY LINKS TO HEART FAILURE}

\section{Heart failure and the scope of the problem}

\begin{abstract}
An estimated 6.5 million American adults are living with heart failure (HF, see Glossary), and its prevalence is projected to rise to over 8 million people by $2030[1,2]$. Prognosis remains poor and mortality unacceptably high: the 5 year survival rate is $\sim 50 \%$ after diagnosis and HF is a contributing cause in 1 of 8 deaths [1]. HF with preserved ejection fraction (HFpEF) currently accounts for approximately $50 \%$ of patients with HF [1], and its prevalence relative to HF with reduced ejection fraction (HFrEF) continues to rise at a rate of $1 \%$ per year [2]. While observational studies have demonstrated comparable risk of death
\end{abstract}

\footnotetext{
*Corresponding Author.
}

Publisher's Disclaimer: This is a PDF file of an unedited manuscript that has been accepted for publication. As a service to our customers we are providing this early version of the manuscript. The manuscript will undergo copyediting, typesetting, and review of the resulting proof before it is published in its final citable form. Please note that during the production process errors may be discovered which could affect the content, and all legal disclaimers that apply to the journal pertain. 
in both HFpEF and HFrEF patients [3], distinct differences in etiology and pathophysiology necessitate unique therapeutic strategies.

\section{HFrEF: Etiology, pathophysiology, and response to pharmacologic therapies}

HFrEF is characterized by reduced left ventricular (LV) contractility, and is often accompanied by LV dilation. While systemic metabolic syndromes can affect outcomes in HFrEF, LV remodeling and systolic dysfunction are generally induced by "damage from within" the myocardium. The myocardial etiology of HFrEF begins with intramyocardial inflammation arising after cardiomyocyte cell death from ischemia, reperfusion injury, infection, toxicity, or genetic mutation [4], and this inflammation promotes the subsequent replacement of myocardium with noncontractile, fibrotic scar. Excessive activation of cardiac beta-adrenergic receptors, the renin-angiotensin-aldosterone system, and the neprilysin pathway all accompany HFrEF; randomized clinical trials have demonstrated that inhibition of all of these pathways improve morbidity and mortality in patients with HFrEF [5].

\section{HFpEF: Etiology, pathophysiology, and response to pharmacologic therapies}

In HFpEF, abnormalities in LV diastolic relaxation and chamber compliance predominate. While EF is preserved, LV contractility is not normal—longitudinal fiber contractility is impaired, and abnormal contractile reserve is present. In contrast to HFrEF, neurohormonal inhibition with angiotensin-converting enzyme inhibitors, beta blockers, and other pharmacologic agents has not improved outcomes in HFpEF patients [6]. These randomized clinical trial results leave the growing HF population with limited therapeutic options. The failure of neurohormonal inhibition strategies in HFpEF may be explained by distinct systemic and myocardial biology in HFpEF, as well as the pathophysiological heterogeneity of HFpEF phenotypes [7]. While the exact etiology of HFpEF is unresolved, extracardiac comorbidities appear to promote systemic inflammation, coronary microvascular endothelial dysfunction, interstitial fibrosis, and impaired cardiomyocyte relaxation; these pathologic processes culminate in LV remodeling and diastolic dysfunction [8]. Common extracardiac risk factors for HFpEF include advanced age, female sex, hypertension, metabolic syndrome, diabetes mellitus, renal dysfunction, and obesity [9].

\section{Inflammation in HFrEF and HFpEF}

Importantly, in both HFrEF and HFpEF, elevated serum proinflammatory cytokines are predictive of worsened clinical outcomes [10-13], which suggests that inflammation may contribute to disease progression in HF patients. The last three decades have seen inflammation emerge as a therapeutic target to mitigate cardiovascular disease. However, strategies employing broad immunosuppression have failed to improve outcomes both after myocardial infarction (MI) [14, 15] and during HF [16]. These observations are consistent with the notion that immune function is also fundamental in orchestrating tissue repair and inflammation resolution (Figure 1) [17]. In 2017, the Canakinumab Anti-Inflammatory Thrombosis Outcome Study (CANTOS), which targeted the inflammatory cytokine, interleukin (IL)-1 $\beta$, demonstrated the merits of immunomodulation (as opposed to 
immunosuppression) in reducing recurrent cardiovascular events through specific inhibition of an inflammatory pathway [18].

Phagocytes within the heart, particularly macrophages, play important roles in maintaining cardiac homeostasis and orchestrating reparative processes after tissue injury. These innate immune cells reside in the myocardium at steady-state and increase in abundance during $\mathrm{HF}$ through systemic mobilization of phagocyte populations from distal sites, including the spleen and bone marrow [19-21]. Macrophages are sensitive to their surroundings and alter their physiology in response to stimuli originating in the myocardium, including myocardial damage-associated molecular patterns (DAMP) [22] and apoptotic cells [23, 24], which trigger pathological inflammatory pathways or tissue reparative processes, respectively. They also adjust their physiology in response to systemic cues, such as those arising during hypertension or aging $[25,26]$. The plasticity of macrophage physiology points to the possibility that macrophage function may precede or promote the pathogenesis of HF. In this review, we discuss recent findings interrogating the role of macrophages in inflammation and its resolution in the context of heart failure, with a specific focus on HFrEF vs HFpEF.

\section{MACROPHAGE HETEROGENEITY IN THE HEART}

Macrophages populate the heart at steady state and maintain tissue homeostasis in healthy human myocardium [27], changing in abundance and phenotype in response to tissue injury or disease. Examination of cardiac tissue specimens from sex-mismatched heart transplant recipients and transcriptional profiling of macrophages led to the identification of two distinct human cardiac macrophage subsets which differ in origin, localization, and inflammatory function [28]. Human cardiac macrophages can be identified as expressing canonical macrophage markers $\mathrm{CD}^{+}{ }^{+}$and $\mathrm{MerTK}^{+}$[29], and are human leukocyte antigen (HLA)-DR ${ }^{\text {high }}$. They are further defined by the expression of the chemokine receptor, C-C chemokine receptor 2 (CCR2) [28], which is required for monocyte egress from the bone marrow [30]. CCR2 ${ }^{-}$macrophages are tissue-resident and maintained through local proliferation, while CCR $2^{+}$macrophages are derived from both monocyte recruitment and local proliferation [28]. In the healthy adult mouse heart, macrophages can be distinguished by the expression of CCR2 and major histocompatibility complex (MHC) II into three main

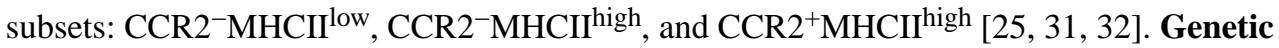
fate mapping, parabiosis, and single-cell transcriptomics of the mouse heart has revealed that $\mathrm{CCR} 2^{-}$macrophages are established early in development from primitive yolk sac and fetal monocyte lineages and are self-maintained into adulthood, while $\mathrm{CCR} 2^{+}$macrophages are replenished by bone marrow-derived blood monocytes [25, 33, 34].

Both macrophage subsets localize to anatomically distinct regions of the heart with CCR2macrophages located within the viable myocardial wall and associated with coronary endothelial cells, while $\mathrm{CCR} 2^{+}$macrophages preferentially occupy trabecular projections of the endocardium and areas containing fibrotic tissue [28, 33]. Divergence in both ontogeny and localization suggests that macrophage subsets have specific functions in the myocardium. For example, CCR2- macrophages localized to the coronary artery in the developing mouse heart are necessary for remodeling of the primitive coronary plexus through secretion of proangiogenic signals, such as insulin like growth factor, leading to 
migration and proliferation of endothelial cells at sites of vascular perfusion [33]; CCR2 ${ }^{-}$ macrophages also populate the atrioventricular node and express connexin-43, which allows electrical coupling with cardiomyocytes and facilitates cardiac electrical conduction [35]. In contrast, $\mathrm{CCR} 2^{+}$macrophages embedded in collagen-rich scar tissue are enriched for genes known to promote fibrosis, hypertrophy, and inflammation [28]. Single-cell transcriptomics of the mouse heart at steady-state have advanced these earlier studies and begun to unravel the complexity underlying resident and recruited macrophages. CCR2- $2^{-} \mathrm{MCII}^{\text {low }}$ macrophages are enriched in genes associated with homeostatic functions, including endocytosis, angiogenesis, and regeneration, while CCR $2^{+}$macrophages are associated with inflammatory processes, respiratory burst, and antigen processing pathways [32]. Importantly, macrophage heterogeneity and plasticity in the heart both at steady-state and after injury extends beyond the traditional M1/M2 macrophage polarization paradigm [32, $36,37]$.

In humans, organ slice cultures and transcriptional profiling have begun to functionally characterize cardiac macrophages with $\mathrm{CCR} 2^{-}$and $\mathrm{CCR} 2^{+}$macrophages enriched in tissue repair and proinflammatory genes, respectively [28]. However, all of these tissues were obtained from patients with either ischemic or dilated cardiomyopathy; therefore, the role of cardiac macrophages in the healthy human heart remains unclear. The differentiation of human induced pluripotent stem cells into macrophages (iPSC-derived macrophages) has emerged as a powerful discovery platform to model disease, perform functional genomic assays, and assess therapeutic potential of human macrophages. iPSC-derived macrophages are genotype-specific, developmentally model tissue resident macrophages, and preserve fundamental interspecies differences, such as long intergenic noncoding RNAs

(LincRNA), which are poorly conserved between humans and mice and have been associated with cardiometabolic disorders [38, 39]. For example, targeted knockdown of macrophage-enriched obesity-associated lincRNA (MacORIS) in human macrophages enhanced interferon (IFN)- $\gamma$-induced signaling pathways, suggesting a repressive role for MacORIS in IFN- $\gamma$ signaling and a cellular mechanism underlying its association with central obesity and related cardiometabolic disorders. Whether cardiac macrophage function in humans is a consequence of ontogeny or environmental niche remains an open question, but differences in origin, localization, and function increase the likelihood that CCR2 ${ }^{-}$and $\mathrm{CCR} 2^{+}$macrophages differentially impact disease development and progression during heart failure as discussed below.

\section{MACROPHAGES INFLAME THE HEART PRIOR TO AND DURING HFrEF}

Acute MI remains a leading cause of HFrEF. Despite medical advances in thrombolytics and percutaneous coronary intervention, which improve patient survival after acute MI, irreversible loss of cardiomyocytes from ischemia and subsequent reperfusion injury initiates a cascade of inflammatory events leading to adverse ventricular remodeling and systolic dysfunction. After permanent occlusion myocardial infarction in the mouse, the initial inflammatory phase (days $0-2$ ) is characterized by a $\sim 50 \%$ reduction of resident macrophages in the infarct region [32]. Local production of chemokines and cytokines, including, CCL2 and granulocyte-macrophage colony-stimulating factor (GM-CSF), by resident $\mathrm{CCR} 2^{+}$macrophages and cardiac fibroblasts attract $\mathrm{CCR} 2^{+}$monocytes to the 
injured heart [37, 40-42], which then differentiate into monocyte-derived macrophages [43]. Recognition of DAMPS, including ATP and self-DNA released by dying cardiomyocytes, provokes proinflammatory responses from macrophages $[22,44,45]$ and contributes to tissue damage. Macrophages also begin to produce bioactive lipid mediators referred to as specialized proresolving mediators (SPM), including resolvins, maresins, and lipoxins, that trigger anti-inflammatory macrophage reprogramming and prime inflammation resolution [46]. Tissue repair (days 3-7) is initiated by the recognition and phagocytosis of dying cardiomyocytes and neutrophils (efferocytosis) by macrophages, which promotes the production of anti-inflammatory and tissue reparative cytokines [24, 47, 48]. Macrophage production of transforming growth factor (TGF) $\beta$ and IL-10 directs cardiac fibroblast migration, proliferation, and collagen expression, leading to deposition of collagen fibers and formation of a mature scar (Figure 2) [49, 50]. Inflammation resolves (days 7-14) following clearance of debris and dead cells and macrophage egress through draining cardiac lymphatics [51, 52]. In humans, post-mortem examination of cardiac, spleen, and bone marrow tissue specimens from 28 patients who died at different time points after acute MI revealed similar recruitment kinetics of monocytes with appearance of $\mathrm{CD} 14^{+}$ monocytes at the infarct border zone during the inflammatory phase (12h-5 days, accompanied by a decrease in $\mathrm{CD} 14^{+}$monocyte numbers in the bone marrow and spleen), followed by accumulation in the infarct core during the proliferative phase (days 5-14) [53]. Over months to years, systemic neuroendocrine activation and compensatory mechanisms, such as LV wall thinning and chamber dilation, are unsustainable and lead to HFrEF.

While the role of macrophages in inflammation and repair after acute MI is relatively welldefined, less is known about their contribution in the chronic setting once HFrEF has been established. Macrophages proliferate during ischemic heart disease [36], and clinically, $\mathrm{CCR} 2^{+}$macrophage abundance is correlated with worsened LV systolic function and chamber dilation in HF patients undergoing LV assist device implantation [28]. Furthermore, $\mathrm{CCR} 2^{+}$macrophages enriched from the hearts of these patients produce robust amounts of IL-1 $\beta$ following exposure to necrotic cardiomyocytes. Prolonged cardiomyocyte apoptosis has been observed in the hearts of patients with ischemic cardiomyopathy [54], and compared to their CCR2 $2^{-}$counterparts, CCR2 ${ }^{+}$macrophages display $\sim 2.5$-fold reduction in apoptotic cell engulfment efficiency [25]. Inability to clear primary apoptotic cardiomyocytes leads to secondary necrosis and the release of DAMPs, which further stimulate proinflammatory responses and collateral tissue injury. In HFrEF patients, systemic levels of both IL-1 $\beta$ and tumor necrosis factor (TNF)- $a$ are increased 2- to 6-fold compared to control subjects and predict worsened outcomes [10,11], suggesting that macrophage-mediated inflammation may have a causal role in HFrEF pathogenesis. Consistent with a role for macrophages in disease progression, blockade of IL- $1 \beta$ with the therapeutic monoclonal antibody canakinumab in a large cohort of patients $(n=10,061)$ with a history of MI and high-sensitivity C-reactive protein levels of over $2 \mathrm{mg} / \mathrm{L}$ lowered the rate of recurrent adverse cardiovascular events over a median follow-up of 3.7 years [18].

Treatment with canakinumab was also related to a significant dose-dependent reduction in hospitalization for heart failure, with a $25 \%$ reduction in patients treated with $300 \mathrm{mg}$ canakinumab every 3 months [55], suggesting that IL-1 $\beta$ plays a causal role in HF progression. IL- $1 \beta$ induces long-lasting transcriptomic and epigenomic reprogramming 
leading to increased cardiac macrophage abundance through myelopoiesis and hyperresponsiveness to inflammatory stimuli in macrophages [56]. In mice with chronic HF, splenic myeloid cells retain expression of inflammatory genes such as TNF- $a$ and IL-6 and adoptive transfer of these cells from chronic HF mice induces HF symptoms in naïve mice [20]. Therefore, increased abundance of cardiac CCR2 $2^{+}$macrophages in the ischemic heart and persistent inflammation reshape the hematopoietic compartment and favor further inflammatory macrophage infiltration into the heart perpetuating adverse remodeling, systolic dysfunction, and HFrEF progression.

Beyond the inflammatory hypothesis, network analyses of biomarkers measured in the blood of HF patients have identified that biological pathways associated with stretch-mediated interactions (N-terminal pro-brain natriuretic peptide, pro-atrial-type natriuretic peptide), proliferation (mitogen-activated protein kinase), and metabolism (protein Kinase B) may be unique to HFrEF compared to HFpEF [57, 58]. Increased wall tension due to left ventricular wall thinning and chamber dilation promotes cardiac macrophage proliferation through strain activation of the mitogen-activated protein kinase pathway [36]. In rat peritoneal macrophages plated on collagen coated wells, exposure to $20 \%$ elongation also stimulated expression of proinflammatory genes, such as IL-6, IL-1 $\beta$, and inducible nitric oxide synthase [59], suggesting that macrophage function adapts to structural changes within the infarcted myocardium. Comparatively little is known about the contribution of macrophage metabolism in the hearts of HFrEF patients, which are characterized by a $\sim 40 \%$ reduction in fatty acid stimulated mitochondrial oxidative phosphorylation compared to controls [60]. Metabolism dictates macrophage function with glycolysis and oxidative phosphorylation promoting pro- and anti-inflammatory function, respectively [61-63]. Recent work in a murine model of MI demonstrated that macrophages resource metabolites from engulfed apoptotic cells to fuel both fatty acid oxidation and the mitochondrial electron transport chain leading to production of anti-inflammatory IL-10 and tissue repair [61]. Whether the diminished oxidative phosphorylation capacity of the myocardium in HF extends to tissue resident macrophages warrants further investigation; macrophage immunometabolism is a candidate mechanism for the compromised energetic status in HFrEF and a promising therapeutic target.

\section{MACROPHAGES STIFFEN THE HEART IN HFpEF}

The incidence of HFpEF is on the rise and will soon become the dominant form of HF. In contrast to HFrEF, HFpEF occurs with minimal loss of cardiomyocytes and manifests as impaired LV relaxation and reduced LV chamber compliance (i.e., diastolic dysfunction), which is characterized by impaired filling of the LV during diastole. Age-associated perturbations in cardiac function and extracardiac comorbidities such as hypertension, obesity, diabetes mellitus, chronic obstructive pulmonary disease, and chronic kidney disease [9], which promote systemic inflammation [64], have been implicated in HFpEF development. Beyond the heart, systemic inflammation manifests as pulmonary hypertension, skeletal muscle weakness and dysfunction, and cardiorenal syndrome. In the heart, inflammation promotes coronary microvascular endothelial dysfunction and increased expression of adhesion molecules [65, 66], which favors myocardial infiltration of macrophages and accumulation of collagen [67]. This disturbs nitric oxide and cyclic 
guanosine monophosphate bioavailability leading to cardiomyocyte hypertrophy and stiffness [68-70]. Together, stiffer cardiomyocytes and macrophage-mediated interstitial fibrosis contribute to diastolic dysfunction.

While inflammation plays a role in both HFrEF and HFpEF [12], biomarker analyses of HF patients have revealed distinct differences in disease pathogenesis, with more prominent roles for inflammation and fibrotic pathways in $\mathrm{HFpEF}$ compared to $\mathrm{HFrEF}$ [57, 58, 71]. Systemic levels of inflammatory cytokines, TNF-a, IL-6, and chemokine, CCL2, are increased 1.3- to 2.4-fold in worsening HFpEF compared to stable disease [12, 13]; these observations suggest that heightened inflammation contributes to clinical deterioration in HFpEF patients. In contrast to HFrEF, less is known about the role of macrophages in HFpEF. In myocardial biopsies from HFpEF patients, cardiac macrophages double in abundance [21] and increase gene expression of profibrotic TGF- $\beta$ (59\% compared to control) [67]; these events appear to contribute to fibroblast activation and excess collagen deposition [21, 67]. Cardiac macrophages also secrete galectin-3, which promotes myocardial fibrosis through myofibroblast activation [72], and phagocytosis induced TGF- $\beta$ expression [73]. In HFpEF patients ( $\mathrm{n}=377)$, a significant increase in plasma galectin-3 levels from median baseline levels of $12.1 \mathrm{ng} / \mathrm{mL}$ to $13.8 \mathrm{ng} / \mathrm{mL}$ over a 12 -month period was associated with worsened clinical outcome and subsequent hospitalization [74], linking even a small change in macrophage-mediated myocardial fibrosis to disease progression. Beyond the heart, flow cytometric quantification of blood from HFpEF patients revealed 2- to 4-fold increases in classical, intermediate, and non-classical monocyte subsets indicative of a chronic state of inflammation during HFpEF [21, 75]. Paradoxically, culture of primary monocytes from healthy individuals with media containing $10 \%$ serum from HFpEF patients for 7 days promoted monocyte differentiation into macrophages expressing IL-10 [75]; this observation suggests that long-lasting, environmental cues in HFpEF patients polarize macrophages towards a fibrogenic phenotype, which favors myocardial collagen deposition and diastolic dysfunction.

Understanding whether macrophage function is a cause or consequence of HFpEF has been challenging due to the limited availability of HFpEF animal models that recapitulate the human disease [76]. In contrast to human HFpEF, where disease progression occurs over months to years, many murine models of HFpEF involve sudden disease onset through surgical or pharmacological intervention. For example, transverse aortic constriction, which is commonly used to model diastolic dysfunction, stimulates an acute inflammatory response that develops progressive deterioration of LV systolic function [77]. However, recent work in a clinically relevant murine model of HFpEF, triggered by hypertension, aldosterone infusion, and renal failure, has revealed important insights into macrophage origin and function in disease development [21]. In HFpEF mice, there was an $~ 2$-fold increase in cardiac macrophage abundance associated with increased production of CCR2 ${ }^{+}$ monocytes in the bone marrow and spleen, leading to increased CCR2-dependent migration to the heart [21]. Inhibition of CCR2 ${ }^{+}$monocyte recruitment during angiotensin-II induced HFpEF using mice deficient in either CCR2 or CCL2 prevented angiotensin-II induced cardiac macrophage expansion and expression of a-smooth muscle actin (a marker of myofibroblast activation and fibrogenesis), and improved diastolic function [78, 79], revealing the importance of monocyte-derived macrophage accumulation and function in 
disease development. Production of IL-10 by cardiac monocyte-derived macrophages expressing high levels of major histocompatibility complex II (MHCII ${ }^{\text {high }}$ ) acted in an autocrine loop to promote profibrotic macrophage polarization characterized by secretion of TGF- $\beta$ and osteopontin, leading to fibroblast activation and collagen deposition, and subsequently myocardial stiffness and diastolic dysfunction (Figure 3) [21]. In HF patients $(\mathrm{n}=460)$, blood levels of osteopontin measured at discharge after hospitalization for acute HF were predicative for all-cause mortality and HF-related rehospitalizations at 18 months in HFpEF but not HFrEF [57], highlighting an important role for cardiac macrophage and fibroblast crosstalk in HFpEF pathogenesis.

Aging, a HFpEF risk factor in humans and HFpEF model in mice, similarly increases the percentage of MHCII ${ }^{\text {high }}$ macrophage density to $\sim 50-60 \%$ of the total macrophage population in the mouse heart, while decreasing the abundance of primitive $\mathrm{MHCII}^{\text {low }}$ macrophages [80], which orchestrate tissue repair and limit adverse remodeling in the neonatal mouse heart [31]. Aging also significantly increases expression of pro-fibrotic genes, such as TGF- $\beta$, two- to four-fold in cardiac macrophages [81], and significantly decreases expression of lipoxgenases (5-, 12-, and 15), the enzymes that are critical for inflammation resolving SPM synthesis [26]. The expansion of MHCII ${ }^{\text {high }}$ cardiac macrophages with aging is due in part to $\mathrm{CCR} 2^{+}$monocyte recruitment as demonstrated by bone marrow reconstitution and parabiosis experiments in mice [80]. Increases in CCR2 ${ }^{+}$ monocyte-dependent expansion of MHCII ${ }^{\text {high }}$ cardiac macrophages (which highly express fibrogenic genes), with a concomitant decrease in $\mathrm{MHCII}^{\text {low }}$ macrophages (which favor matrix breakdown) [21], during physiological aging in mice may place the aged heart at risk for the development of HFpEF. It is unclear whether this translates to the human heart during senescence; however the human equivalent of mouse MHCII ${ }^{\text {high }}$ cardiac macrophages, CCR2 ${ }^{+} \mathrm{HLA}-\mathrm{DR}{ }^{\text {high }}$ macrophages, are enriched in genes known to promote fibrosis, such as amphiregulin, pentraxin 3, and oncostatin M [28]. Furthermore, agedependent changes in innate immune activation and myocardial fibrosis have been reported in human cardiomyopathies. Examination of cardiac tissue specimens from pediatric $(n=31)$ and adult $(n=34)$ patients with dilated cardiomyopathies revealed minimal interstitial fibrosis in pediatric hearts compared to adults and selective upregulation of proinflammatory genes, including IL-1 $\beta$ and CCL2, in adult hearts [82]. Consequently, changes in macrophage subsets in the aged heart may precede and promote the development of HFpEF.

Additional mechanistic studies are required to interrogate upstream signaling pathways regulating macrophages and how macrophage function differentially impacts disease development and progression in HFpEF and HFrEF. For example, macrophage production of IL-10 is beneficial in tissue repair and inflammation resolution following acute injury, preventing HFrEF after MI [61, 83], but is deleterious in a chronic disease setting, promoting myocardial fibrosis and diastolic dysfunction in HFpEF [21]. In rats with HFrEF, whole heart IL-10 gene expression is significantly reduced by $\sim 70 \%$ at 16 weeks after myocardial infarction compared to sham controls [84]. In contrast, in mice with HFpEF after physiological aging to 30 months, whole heart IL-10 gene expression is significantly increased $~ 9$-fold compared to the hearts of 8 week old mice [21], suggesting that sustained expression of IL-10 may favor profibrotic effects. Consistent with this dual role for IL-10, short-term overexpression of doxycycline induced lung-specific human IL-10 in mice for 5 
days attenuated LPS-induced acute inflammation [85], while longer-term overexpression for 1 month promoted lung fibrosis [86]. The availability of inducible $C r e$ recombinase transgenic mice, which enable temporal deletion of proteins, may facilitate our understanding on the dual roles of proteins in physiological and pathological inflammation after establishment of chronic HF. That the same pathways may be beneficial in HFrEF but pathologic in HFpEF has important implications in designing therapeutic strategies to limit disease progression in HF of diverse etiologies.

\section{TARGETING MACROPHAGES IN HEART FAILURE: BLOCKING THE BAD, PROMOTING THE GOOD}

The correlation between macrophage abundance/function and disease progression in human HF supports the development of therapeutics that inhibit recruitment and neutralize damaging inflammatory functions of macrophages to promote recovery of the failing heart. In a human pilot study and murine models, administration of Anakinra, the recombinant form of the naturally occurring IL-1 receptor antagonist, limited adverse remodeling and preserved LV systolic function after acute MI [87-89], highlighting the importance of early inflammatory events establishing the trajectory towards HFrEF. Similarly, treatment of 12 HFpEF patients with Anakinra for 28 days attenuated both systemic inflammation and disease symptoms, measured as significant changes in plasma C-reactive protein levels (74\% reduction) and aerobic exercise capacity $(+1.2 \mathrm{ml} / \mathrm{kg} / \mathrm{min})$, respectively [90]. While the results in HFpEF are promising, the multifactorial etiology and phenotypic diversity in HFpEF necessitates targeting inflammation within personalized therapeutic strategies that also target patient-specific comorbidities [91, 92], including selection of the right inflammatory target. In clinical trials of HF patients with New York Heart Association (NYHA) class II-IV symptoms, targeting TNF- $a$ with either etanercept $(n=2,048)$, a soluble TNF receptor fusion protein, or infliximab $(n=150)$, a monoclonal antibody, anti-TNFa therapies resulted in no clinical benefit and in some cases, increased the risk of death over the course of 24 to 28 weeks $[93,94]$. The lack of clinical benefit may have been due to excessive TNF- $a$ antagonism or loss of TNF- $a$-dependent cardioprotective effects (preventing apoptosis in stressed myocytes) [93, 94]. Caution is also needed when targeting macrophage function to limit the incidence of adverse side effects, such as increased risk for infection or tumorigenesis. Alternative strategies, such as nanoparticles loaded with therapeutic cargo, that target inflammatory monocyte recruitment to the injured heart may improve tissue repair [95, 96], while preserving host defense functions to limit opportunistic infections.

In addition to blocking inflammatory macrophage recruitment and function, selective activation of macrophage pathways involved in inflammation resolution may confer clinical benefits in HF patients. For example, engulfment of apoptotic cells by macrophages imprints a distinct anti-inflammatory phenotype [97]. Using the anti-inflammatory properties of apoptotic cells to counteract chronic inflammation present in HF, patients with NYHA class II-IV symptoms $(n=2,426)$ were enrolled in the ACCLAIM trial and treated with their own blood that had been stressed ex vivo with ultraviolet light to induce apoptosis and immediately administered back to the same patient by intramuscular injection [98]. 
Immunomodulation with the patient's own apoptotic cells was associated with $26 \%$ and $39 \%$ significant reductions in all-cause mortality and cardiovascular hospital admission over an average follow-up of 10.2 months in HF patients without a history of MI and with more mild HF (NYHA class II) symptoms, respectively [98]. While non-specific immunomodulation demonstrated therapeutic promise in the ACCLAIM trial, additional macrophage-targeted approaches are currently under development, including cardiosphere-derived cells (CDC), heart cell products with anti-fibrotic and anti-inflammatory properties. In a rat model of HFpEF, administration of CDCs for 4 weeks after 7 weeks of high-salt diet feeding of Dahl salt-sensitive rats reversed systemic inflammation and cardiac fibrosis and resolved diastolic dysfunction [99]. CDCs mediate their cardioprotective effect through macrophages, as systemic depletion of macrophages with clodronate abolished the CDC-mediated 3-fold reduction in infarct size in a rat model of MI [100]. The expansion of these therapeutic strategies and others that selectively target macrophages hold promise in reducing morbidity and mortality of HF patients, while limiting adverse events.

\section{CONCLUDING REMARKS AND FUTURE PERSPECTIVES}

There is a wealth of knowledge on macrophages and inflammation from both human and animal HF studies but we still lack a clear understanding of whether macrophage function is a cause or consequence in human disease development, and even less is known about the role of these cells after chronic HF has been established (see Outstanding Questions). Animal models have advanced our understanding of the complexity and plasticity underlying resident and recruited cardiac macrophage biology in both steady-state and chronic HF and have identified causal roles for macrophages in HF pathogenesis; however, it remains unclear whether these findings will translate to human disease. Application of cutting-edge tools, including single cell RNA sequencing of cardiac tissue specimens and human induced pluripotent stem cell-derived macrophages, will enable investigation into the complexity and plasticity underlying human cardiac macrophages at both steady-state and during chronic $\mathrm{HF}$ and stimulate research into known genetic mutations that are associated with HF but lack a suitable murine model. Further investigation defining the crosstalk between macrophages and other cardiac resident cells, including fibroblasts and cardiomyocytes, and the influence of comorbidities and risk factors on macrophage heterogeneity and function are also needed to delineate the role of macrophages in chronic $\mathrm{HF}$ and inform therapeutic strategies. For example, proinflammatory CCR2 ${ }^{+}$macrophage abundance is increased in the heart with age and is a plausible mechanism for the exaggerated inflammatory responses and worsened outcomes in older HF patients; as such, selective therapeutic targeting of CCR2 ${ }^{+}$macrophages may confer clinical benefits to elderly patients. Ultimately, a balanced approach with both animal and human models facilitates a better understanding of HF pathogenesis in humans, informing novel therapeutic strategies that promote beneficial clinical outcomes in humans.

\section{GLOSSARY}

\section{Cardiosphere-Derived Cell}

Heterogenous mix of cells expanded from cardiac tissue that exhibit multilineage potential 


\section{Diastolic Dysfunction}

Impaired LV relaxation and reduced chamber compliance characterized by impaired filling of the LV during diastole

\section{Efferocytosis}

Removal of apoptotic cells by phagocytic cells

\section{Heart Failure}

Chronic and progressive inability of the heart to pump an adequate supply of blood to meet the body's demands in blood and oxygen

\section{Genetic Fate Mapping}

Method to define the relationship between the embryonic origin of an individual cell and its progeny at later stages of development

\section{Immunomodulation}

Modulation of immune responses (induced, amplified, attenuated, or inhibited) to achieve a desired therapeutic effect

\section{Induced Pluripotent Stem Cell-Derived Macrophage}

Differentiation of human induced pluripotent stem cell to produce myeloid progenitors followed by culture with macrophage colony-stimulating factor for directed differentiation into mature macrophages

\section{Interstitial Fibrosis}

Thickening and scarring of connective tissue in the heart

\section{Long Intergenic Noncoding RNA}

RNA transcripts longer than 200 nucleotides that are not translated into protein

\section{Myelopoiesis}

Development of myeloid cells, including monocytes, in the bone marrow

\section{Parabiosis}

Anatomical joining of two animals to study recruitment and turnover of macrophage populations in tissues

\section{Secondary Necrosis}

Apoptotic cells not cleared in an efficient or timely manner become necrotic, characterized by loss of membrane integrity and release of cellular components

\section{Single-cell Transcriptomics}

Method to define expression level of hundreds to thousands of genes in an individual cell within a heterogenous population

\section{Specialized Proresolving Mediators}

Class of lipid mediators enzymatically derived by metabolism of polyunsaturated fatty acids

\section{Trabecular (trabeculae) Projections}


Finger-like bundles of myocardial tissue that extend into the RV and LV chambers

\section{References}

1. Benjamin EJ et al. (2018) Heart Disease and Stroke Statistics-2018 Update: A Report From the American Heart Association. Circulation 137 (12), e67-e492. [PubMed: 29386200]

2. Heidenreich PA et al. (2013) Forecasting the impact of heart failure in the United States: a policy statement from the American Heart Association. Circ Heart Fail 6 (3), 606-19. [PubMed: 23616602]

3. Lee DS et al. (2009) Relation of disease pathogenesis and risk factors to heart failure with preserved or reduced ejection fraction: insights from the framingham heart study of the national heart, lung, and blood institute. Circulation 119 (24), 3070-7. [PubMed: 19506115]

4. Chiong $\mathrm{M}$ et al. (2011) Cardiomyocyte death: mechanisms and translational implications. Cell Death Dis 2, e244. [PubMed: 22190003]

5. Yancy CW et al. (2017) 2017 ACC/AHA/HFSA Focused Update of the 2013 ACCF/AHA Guideline for the Management of Heart Failure: A Report of the American College of Cardiology/American Heart Association Task Force on Clinical Practice Guidelines and the Heart Failure Society of America. J Card Fail 23 (8), 628-651. [PubMed: 28461259]

6. Cleland JG et al. (2006) The perindopril in elderly people with chronic heart failure (PEP-CHF) study. Eur Heart J 27 (19), 2338-45. [PubMed: 16963472]

7. Shah SJ et al. (2015) Phenomapping for novel classification of heart failure with preserved ejection fraction. Circulation 131 (3), 269-79. [PubMed: 25398313]

8. Paulus WJ and Tschope C (2013) A novel paradigm for heart failure with preserved ejection fraction: comorbidities drive myocardial dysfunction and remodeling through coronary microvascular endothelial inflammation. J Am Coll Cardiol 62 (4), 263-71. [PubMed: 23684677]

9. Dunlay SM et al. (2017) Epidemiology of heart failure with preserved ejection fraction. Nat Rev Cardiol 14 (10), 591-602. [PubMed: 28492288]

10. Deswal A et al. (2001) Cytokines and cytokine receptors in advanced heart failure: an analysis of the cytokine database from the Vesnarinone trial (VEST). Circulation 103 (16), 2055-9. [PubMed: 11319194]

11. Testa M et al. (1996) Circulating levels of cytokines and their endogenous modulators in patients with mild to severe congestive heart failure due to coronary artery disease or hypertension. J Am Coll Cardiol 28 (4), 964-71. [PubMed: 8837575]

12. Collier $P$ et al. (2011) Can emerging biomarkers of myocardial remodelling identify asymptomatic hypertensive patients at risk for diastolic dysfunction and diastolic heart failure? Eur J Heart Fail 13 (10), 1087-95. [PubMed: 21719449]

13. Abernethy A et al. (2018) Pro-Inflammatory Biomarkers in Stable Versus Acutely Decompensated Heart Failure With Preserved Ejection Fraction. J Am Heart Assoc 7 (8).

14. Baran KW et al. (2001) Double-blind, randomized trial of an anti-CD18 antibody in conjunction with recombinant tissue plasminogen activator for acute myocardial infarction: limitation of myocardial infarction following thrombolysis in acute myocardial infarction (LIMIT AMI) study. Circulation 104 (23), 2778-83. [PubMed: 11733394]

15. Gislason GH et al. (2006) Risk of death or reinfarction associated with the use of selective cyclooxygenase- 2 inhibitors and nonselective nonsteroidal antiinflammatory drugs after acute myocardial infarction. Circulation 113 (25), 2906-13. [PubMed: 16785336]

16. McNamara DM et al. (2001) Controlled trial of intravenous immune globulin in recent-onset dilated cardiomyopathy. Circulation 103 (18), 2254-9. [PubMed: 11342473]

17. Serhan CN and Savill J (2005) Resolution of inflammation: the beginning programs the end. Nat Immunol 6 (12), 1191-7. [PubMed: 16369558]

18. Ridker PM et al. (2017) Antiinflammatory Therapy with Canakinumab for Atherosclerotic Disease. N Engl J Med 377 (12), 1119-1131. [PubMed: 28845751] 
19. Kim EJ et al. (2014) Metabolic activity of the spleen and bone marrow in patients with acute myocardial infarction evaluated by $18 \mathrm{f}$-fluorodeoxyglucose positron emission tomograpic imaging. Circ Cardiovasc Imaging 7 (3), 454-60. [PubMed: 24488982]

20. Ismahil MA et al. (2014) Remodeling of the mononuclear phagocyte network underlies chronic inflammation and disease progression in heart failure: critical importance of the cardiosplenic axis. Circ Res 114 (2), 266-82. [PubMed: 24186967]

21. Hulsmans M et al. (2018) Cardiac macrophages promote diastolic dysfunction. J Exp Med 215 (2), 423-440. [PubMed: 29339450]

22. King KR et al. (2017) IRF3 and type I interferons fuel a fatal response to myocardial infarction. Nat Med 23 (12), 1481-1487. [PubMed: 29106401]

23. Wan E et al. (2013) Enhanced efferocytosis of apoptotic cardiomyocytes through myeloidepithelial-reproductive tyrosine kinase links acute inflammation resolution to cardiac repair after infarction. Circ Res 113 (8), 1004-12. [PubMed: 23836795]

24. Howangyin KY et al. (2016) Myeloid-Epithelial-Reproductive Receptor Tyrosine Kinase and Milk Fat Globule Epidermal Growth Factor 8 Coordinately Improve Remodeling After Myocardial Infarction via Local Delivery of Vascular Endothelial Growth Factor. Circulation 133 (9), 826-39. [PubMed: 26819373]

25. Epelman S et al. (2014) Embryonic and adult-derived resident cardiac macrophages are maintained through distinct mechanisms at steady state and during inflammation. Immunity 40 (1), 91-104. [PubMed: 24439267]

26. Halade GV et al. (2016) Aging dysregulates D- and E-series resolvins to modulate cardiosplenic and cardiorenal network following myocardial infarction. Aging (Albany NY) 8 (11), 2611-2634. [PubMed: 27777380]

27. Devaux B et al. (1997) Upregulation of cell adhesion molecules and the presence of low grade inflammation in human chronic heart failure. Eur Heart J 18 (3), 470-9. [PubMed: 9076385]

28. Bajpai $\mathrm{G}$ et al. (2018) The human heart contains distinct macrophage subsets with divergent origins and functions. Nat Med 24 (8), 1234-1245. [PubMed: 29892064]

29. Gautier EL et al. (2012) Gene-expression profiles and transcriptional regulatory pathways that underlie the identity and diversity of mouse tissue macrophages. Nat Immunol 13 (11), 1118-28. [PubMed: 23023392]

30. Tsou CL et al. (2007) Critical roles for CCR2 and MCP-3 in monocyte mobilization from bone marrow and recruitment to inflammatory sites. J Clin Invest 117 (4), 902-9. [PubMed: 17364026]

31. Lavine KJ et al. (2014) Distinct macrophage lineages contribute to disparate patterns of cardiac recovery and remodeling in the neonatal and adult heart. Proc Natl Acad Sci U S A 111 (45), 16029-34. [PubMed: 25349429]

32. Dick SA et al. (2019) Self-renewing resident cardiac macrophages limit adverse remodeling following myocardial infarction. Nat Immunol 20 (1), 29-39. [PubMed: 30538339]

33. Leid J et al. (2016) Primitive Embryonic Macrophages are Required for Coronary Development and Maturation. Circ Res 118 (10), 1498-511. [PubMed: 27009605]

34. Heidt $\mathrm{T}$ et al. (2014) Differential contribution of monocytes to heart macrophages in steady-state and after myocardial infarction. Circ Res 115 (2), 284-95. [PubMed: 24786973]

35. Hulsmans M et al. (2017) Macrophages Facilitate Electrical Conduction in the Heart. Cell 169 (3), 510-522 e20. [PubMed: 28431249]

36. Sager HB et al. (2016) Proliferation and Recruitment Contribute to Myocardial Macrophage Expansion in Chronic Heart Failure. Circ Res 119 (7), 853-64. [PubMed: 27444755]

37. Bajpai G et al. (2018) Tissue Resident CCR2- and CCR2+ Cardiac Macrophages Differentially Orchestrate Monocyte Recruitment and Fate Specification Following Myocardial Injury. Circ Res.

38. Zhang H and Reilly MP (2017) Human Induced Pluripotent Stem Cell-Derived Macrophages for Unraveling Human Macrophage Biology. Arterioscler Thromb Vasc Biol 37 (11), 2000-2006. [PubMed: 28982665]

39. Zhang H et al. (2017) Deep RNA Sequencing Uncovers a Repertoire of Human Macrophage Long Intergenic Noncoding RNAs Modulated by Macrophage Activation and Associated With Cardiometabolic Diseases. J Am Heart Assoc 6 (11). 
40. Wu L et al. (2014) Cardiac fibroblasts mediate IL-17A-driven inflammatory dilated cardiomyopathy. J Exp Med 211 (7), 1449-64. [PubMed: 24935258]

41. Chen G et al. (2018) Sca-1(+) cardiac fibroblasts promote development of heart failure. Eur J Immunol 48 (9), 1522-1538. [PubMed: 29953616]

42. Anzai A et al. (2017) The infarcted myocardium solicits GM-CSF for the detrimental oversupply of inflammatory leukocytes. J Exp Med 214 (11), 3293-3310. [PubMed: 28978634]

43. Hilgendorf I et al. (2014) Ly-6Chigh monocytes depend on Nr4a1 to balance both inflammatory and reparative phases in the infarcted myocardium. Circ Res 114 (10), 1611-22. [PubMed: 24625784]

44. Mezzaroma E et al. (2011) The inflammasome promotes adverse cardiac remodeling following acute myocardial infarction in the mouse. Proc Natl Acad Sci U S A 108 (49), 19725-30. [PubMed: 22106299]

45. Cao DJ et al. (2018) Cytosolic DNA Sensing Promotes Macrophage Transformation and Governs Myocardial Ischemic Injury. Circulation 137 (24), 2613-2634. [PubMed: 29437120]

46. Halade GV et al. (2018) Splenic leukocytes define the resolution of inflammation in heart failure. Sci Signal 11 (520).

47. Cai B et al. (2016) MerTK cleavage limits proresolving mediator biosynthesis and exacerbates tissue inflammation. Proc Natl Acad Sci U S A 113 (23), 6526-31. [PubMed: 27199481]

48. DeBerge M et al. (2017) MerTK Cleavage on Resident Cardiac Macrophages Compromises Repair After Myocardial Ischemia Reperfusion Injury. Circ Res 121 (8), 930-940. [PubMed: 28851810]

49. Shiraishi M et al. (2016) Alternatively activated macrophages determine repair of the infarcted adult murine heart. J Clin Invest 126 (6), 2151-66. [PubMed: 27140396]

50. Jung $\mathrm{M}$ et al. (2017) IL-10 improves cardiac remodeling after myocardial infarction by stimulating M2 macrophage polarization and fibroblast activation. Basic Res Cardiol 112 (3), 33. [PubMed: 28439731]

51. Leuschner F et al. (2012) Rapid monocyte kinetics in acute myocardial infarction are sustained by extramedullary monocytopoiesis. J Exp Med 209 (1), 123-37. [PubMed: 22213805]

52. Klotz L et al. (2015) Cardiac lymphatics are heterogeneous in origin and respond to injury. Nature 522 (7554), 62-7. [PubMed: 25992544]

53. van der Laan AM et al. (2014) Monocyte subset accumulation in the human heart following acute myocardial infarction and the role of the spleen as monocyte reservoir. Eur Heart J 35 (6), 376-85. [PubMed: 23966310]

54. Narula J et al. (1996) Apoptosis in myocytes in end-stage heart failure. N Engl J Med 335 (16), 1182-9. [PubMed: 8815940]

55. Everett BM et al. (2018) Anti-Inflammatory Therapy with Canakinumab for the Prevention of Hospitalization for Heart Failure. Circulation.

56. Christ A et al. (2018) Western Diet Triggers NLRP3-Dependent Innate Immune Reprogramming. Cell 172 (1-2), 162-175 e14. [PubMed: 29328911]

57. Tromp J et al. (2017) Biomarker Profiles in Heart Failure Patients With Preserved and Reduced Ejection Fraction. J Am Heart Assoc 6 (4).

58. Tromp J et al. (2018) Identifying Pathophysiological Mechanisms in Heart Failure With Reduced Versus Preserved Ejection Fraction. J Am Coll Cardiol 72 (10), 1081-1090. [PubMed: 30165978]

59. Wehner S et al. (2010) Mechanical strain and TLR4 synergistically induce cell-specific inflammatory gene expression in intestinal smooth muscle cells and peritoneal macrophages. Am J Physiol Gastrointest Liver Physiol 299 (5), G1187-97. [PubMed: 20829523]

60. Stride N et al. (2013) Decreased mitochondrial oxidative phosphorylation capacity in the human heart with left ventricular systolic dysfunction. Eur J Heart Fail 15 (2), 150-7. [PubMed: 23115323]

61. Zhang S et al. (2018) Efferocytosis Fuels Requirements of Fatty Acid Oxidation and the Electron Transport Chain to Polarize Macrophages for Tissue Repair. Cell Metab.

62. Tannahill GM et al. (2013) Succinate is an inflammatory signal that induces IL-1beta through HIF-1alpha. Nature 496 (7444), 238-42. [PubMed: 23535595] 
63. Mouton AJ et al. (2018) Mapping macrophage polarization over the myocardial infarction time continuum. Basic Res Cardiol 113 (4), 26. [PubMed: 29868933]

64. Santhanakrishnan R et al. (2012) Growth differentiation factor 15, ST2, high-sensitivity troponin T, and N-terminal pro brain natriuretic peptide in heart failure with preserved vs. reduced ejection fraction. Eur J Heart Fail 14 (12), 1338-47. [PubMed: 22869458]

65. Franssen C et al. (2016) Myocardial Microvascular Inflammatory Endothelial Activation in Heart Failure With Preserved Ejection Fraction. JACC Heart Fail 4 (4), 312-24. [PubMed: 26682792]

66. Shah SJ et al. (2018) Prevalence and correlates of coronary microvascular dysfunction in heart failure with preserved ejection fraction: PROMIS-HFpEF. Eur Heart J 39 (37), 3439-3450. [PubMed: 30165580]

67. Westermann D et al. (2011) Cardiac inflammation contributes to changes in the extracellular matrix in patients with heart failure and normal ejection fraction. Circ Heart Fail 4 (1), 44-52. [PubMed: 21075869]

68. van Heerebeek L et al. (2012) Low myocardial protein kinase G activity in heart failure with preserved ejection fraction. Circulation 126 (7), 830-9. [PubMed: 22806632]

69. van Heerebeek L et al. (2008) Diastolic stiffness of the failing diabetic heart: importance of fibrosis, advanced glycation end products, and myocyte resting tension. Circulation 117 (1), 4351. [PubMed: 18071071]

70. Zile MR et al. (2015) Myocardial stiffness in patients with heart failure and a preserved ejection fraction: contributions of collagen and titin. Circulation 131 (14), 1247-59. [PubMed: 25637629]

71. Sanders-van Wijk S et al. (2015) Circulating biomarkers of distinct pathophysiological pathways in heart failure with preserved vs. reduced left ventricular ejection fraction. Eur J Heart Fail 17 (10), 1006-14. [PubMed: 26472682]

72. Gonzalez GE et al. (2016) Cardiac-deleterious role of galectin-3 in chronic angiotensin II-induced hypertension. Am J Physiol Heart Circ Physiol 311 (5), H1287-H1296. [PubMed: 27496875]

73. Mukaro VR et al. (2013) Lectins offer new perspectives in the development of macrophagetargeted therapies for COPD/emphysema. PLoS One 8 (2), e56147. [PubMed: 23441163]

74. Edelmann F et al. (2015) Galectin-3 in patients with heart failure with preserved ejection fraction: results from the Aldo-DHF trial. Eur J Heart Fail 17 (2), 214-23. [PubMed: 25418979]

75. Glezeva $\mathrm{N}$ et al. (2015) Exaggerated inflammation and monocytosis associate with diastolic dysfunction in heart failure with preserved ejection fraction: evidence of M2 macrophage activation in disease pathogenesis. J Card Fail 21 (2), 167-77. [PubMed: 25459685]

76. Valero-Munoz M et al. (2017) Murine Models of Heart Failure with Preserved Ejection Fraction: a "Fishing Expedition". JACC Basic Transl Sci 2 (6), 770-789. [PubMed: 29333506]

77. Patel B et al. (2018) CCR2(+) Monocyte-Derived Infiltrating Macrophages Are Required for Adverse Cardiac Remodeling During Pressure Overload. JACC Basic Transl Sci 3 (2), 230-244. [PubMed: 30062209]

78. Haudek SB et al. (2010) Monocytic fibroblast precursors mediate fibrosis in angiotensin-II-induced cardiac hypertrophy. J Mol Cell Cardiol 49 (3), 499-507. [PubMed: 20488188]

79. Xu J et al. (2011) CCR2 mediates the uptake of bone marrow-derived fibroblast precursors in angiotensin II-induced cardiac fibrosis. Am J Physiol Heart Circ Physiol 301 (2), H538-47. [PubMed: 21572015]

80. Molawi K et al. (2014) Progressive replacement of embryo-derived cardiac macrophages with age. J Exp Med 211 (11), 2151-8. [PubMed: 25245760]

81. Pinto AR et al. (2014) Age-related changes in tissue macrophages precede cardiac functional impairment. Aging (Albany NY) 6 (5), 399-413. [PubMed: 24861132]

82. Patel MD et al. (2017) Pediatric and adult dilated cardiomyopathy represent distinct pathological entities. JCI Insight 2 (14).

83. Krishnamurthy P et al. (2009) IL-10 inhibits inflammation and attenuates left ventricular remodeling after myocardial infarction via activation of STAT3 and suppression of HuR. Circ Res 104 (2), e9-18. [PubMed: 19096025]

84. Kaur K et al. (2006) Significance of changes in TNF-alpha and IL-10 levels in the progression of heart failure subsequent to myocardial infarction. Am J Physiol Heart Circ Physiol 291 (1), H10613. [PubMed: 16461369] 
85. Spight D et al. (2005) Immunoregulatory effects of regulated, lung-targeted expression of IL-10 in vivo. Am J Physiol Lung Cell Mol Physiol 288 (2), L251-65. [PubMed: 15466252]

86. Sun L et al. (2011) New concepts of IL-10-induced lung fibrosis: fibrocyte recruitment and M2 activation in a CCL2/CCR2 axis. Am J Physiol Lung Cell Mol Physiol 300 (3), L341-53. [PubMed: 21131395]

87. Abbate A et al. (2010) Interleukin-1 blockade with anakinra to prevent adverse cardiac remodeling after acute myocardial infarction (Virginia Commonwealth University Anakinra Remodeling Trial [VCUART] Pilot study). Am J Cardiol 105 (10), 1371-1377 e1. [PubMed: 20451681]

88. Toldo $S$ et al. (2014) Interleukin-1beta blockade improves left ventricular systolic/diastolic function and restores contractility reserve in severe ischemic cardiomyopathy in the mouse. $\mathrm{J}$ Cardiovasc Pharmacol 64 (1), 1-6. [PubMed: 25006675]

89. Sager HB et al. (2015) Targeting Interleukin-1beta Reduces Leukocyte Production After Acute Myocardial Infarction. Circulation 132 (20), 1880-90. [PubMed: 26358260]

90. Van Tassell BW et al. (2014) Effects of interleukin-1 blockade with anakinra on aerobic exercise capacity in patients with heart failure and preserved ejection fraction (from the D-HART pilot study). Am J Cardiol 113 (2), 321-327. [PubMed: 24262762]

91. Shah SJ et al. (2016) Phenotype-Specific Treatment of Heart Failure With Preserved Ejection Fraction: A Multiorgan Roadmap. Circulation 134 (1), 73-90. [PubMed: 27358439]

92. Lai YC et al. (2016) SIRT3-AMP-Activated Protein Kinase Activation by Nitrite and Metformin Improves Hyperglycemia and Normalizes Pulmonary Hypertension Associated With Heart Failure With Preserved Ejection Fraction. Circulation 133 (8), 717-31. [PubMed: 26813102]

93. Chung ES et al. (2003) Randomized, double-blind, placebo-controlled, pilot trial of infliximab, a chimeric monoclonal antibody to tumor necrosis factor-alpha, in patients with moderate-to-severe heart failure: results of the anti-TNF Therapy Against Congestive Heart Failure (ATTACH) trial. Circulation 107 (25), 3133-40. [PubMed: 12796126]

94. Mann DL et al. (2004) Targeted anticytokine therapy in patients with chronic heart failure: results of the Randomized Etanercept Worldwide Evaluation (RENEWAL). Circulation 109 (13), 1594602. [PubMed: 15023878]

95. Leuschner F et al. (2011) Therapeutic siRNA silencing in inflammatory monocytes in mice. Nat Biotechnol 29 (11), 1005-10. [PubMed: 21983520]

96. Getts DR et al. (2014) Therapeutic inflammatory monocyte modulation using immune-modifying microparticles. Sci Transl Med 6 (219), 219 ra7.

97. Gonzalez A et al. (2017) Phagocytosis imprints heterogeneity in tissue-resident macrophages. J Exp Med 214 (5), 1281-1296. [PubMed: 28432199]

98. Torre-Amione G et al. (2008) Results of a non-specific immunomodulation therapy in chronic heart failure (ACCLAIM trial): a placebo-controlled randomised trial. Lancet 371 (9608), 228-36. [PubMed: 18207018]

99. Gallet $\mathrm{R}$ et al. (2016) Cardiosphere-derived cells reverse heart failure with preserved ejection fraction (HFpEF) in rats by decreasing fibrosis and inflammation. JACC Basic Transl Sci 1 (1-2), 14-28. [PubMed: 27104217]

100. de Couto G et al. (2015) Macrophages mediate cardioprotective cellular postconditioning in acute myocardial infarction. J Clin Invest 125 (8), 3147-62. [PubMed: 26214527] 


\section{HIGHLIGHTS}

- $\quad$ The increasing prevalence of heart failure and heart failure with preserved ejection fraction, which lacks adequate therapeutics, necessitates a better understanding of the mechanisms of disease pathogenesis.

- Inflammation is correlated with adverse clinical outcomes in HF patients. While results from clinical trials with broad immunosuppression have failed to show effect, specific targeting of proinflammatory cytokines has conferred clinical benefits.

- $\quad$ Phagocytes, including macrophages, regulate inflammatory and reparative processes. Distinct subsets with inflammatory function were recently identified in the human heart and may be linked to HF development and progression. 


\section{CLINICIAN'S CORNER}

- Inflammation significantly contributes to pathological cardiac remodeling in both HFrEF and HFpEF. However, clinical trials of broad immunosuppression have failed to improve outcomes for these diseases, which indicate the dual roles of immune function in both the generation and resolution of inflammation.

- $\quad$ Selective inhibition of interleukin (IL)-1 $1 \beta$ reduced cardiac events in the Canakinumab Anti-Inflammatory Thrombosis Outcome Study (CANTOS), which demonstrates that specific, targeted approaches to immune modulation hold promise in heart failure. However, treatment with canakinumab was also associated with a higher incidence of fatal infection.

- Macrophages are important immune modulators that participate both in the initiation and in the resolution of inflammation in the heart after acute injury. Macrophage contributions to chronic heart failure are less well understood, but current data suggest a greater pro-inflammatory than anti-inflammatory role overall.

- Some cytokine and macrophage signaling pathways appear to be beneficial in $\mathrm{HFrEF}$ but pathologic in $\mathrm{HFpEF}$, which carries important implications in designing therapeutic strategies.

- Immunomodulatory therapies for chronic heart failure that inhibit the proinflammatory and activate the anti-inflammatory actions of macrophages are being actively pursued in basic and translational research programs. Immunomodulatory therapies that minimize off-target effects, such as immunosuppression, hold the greatest therapeutic promise. 


\section{OUTSTANDING QUESTIONS BOX}

- Are distinct macrophage subsets linked to HF development and progression? In humans, the subsets and function of macrophages present in healthy hearts and whether there are disease-specific changes in macrophages during $\mathrm{HFrEF}$ or HFpEF remains unclear.

- What are the role of macrophages in chronic HF? Transgenic mice that permit temporal deletion of proteins will shed light on signaling pathways that are important in disease maintenance and progression after HF is established.

- What is the role of immunometabolism in HF? Metabolic reprogramming regulates macrophage phenotype and may be linked to clinical outcomes in HF.

- $\quad$ Can we directly target macrophages during HF? Nanoparticle technologies enable targeted delivery of therapeutics (i.e. drugs, gene editing) to macrophages to limit side effects and maximize therapeutic efficacy.

- Do similar immune mechanisms underlie other etiologies (i.e. genetic) of HF? Application of cutting-edge tools, including single cell RNA sequencing and human induced pluripotent stem cell-derived macrophages, will stimulate investigation into human cardiomyopathies that lack suitable animal models. 


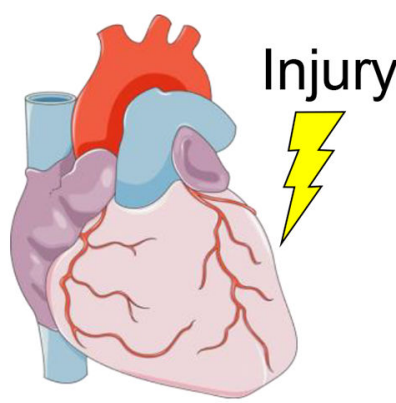

\begin{tabular}{|c|c|}
\hline Cardiomyocyte & $\Rightarrow$ \\
\hline $\begin{array}{l}\text { Apoptotic } \\
\text { cardiomyocyte }\end{array}$ & \\
\hline CCR2- MФ & \\
\hline $\mathrm{CCR}^{+}{ }^{+} \mathrm{M} \Phi$ & \\
\hline CCR2 ${ }^{+}$Monocyte & \\
\hline Cytokine & \\
\hline Fibroblast & \\
\hline Collagen & \\
\hline
\end{tabular}

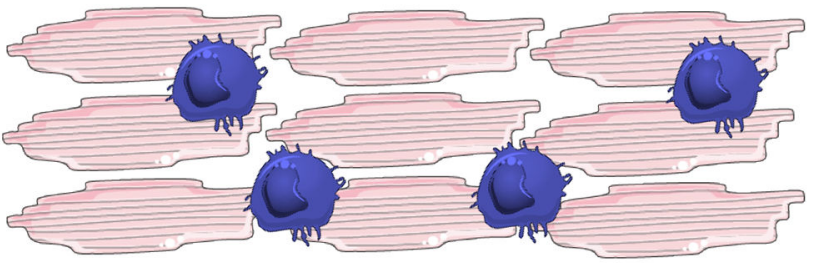

\section{Inflammation $\downarrow$}
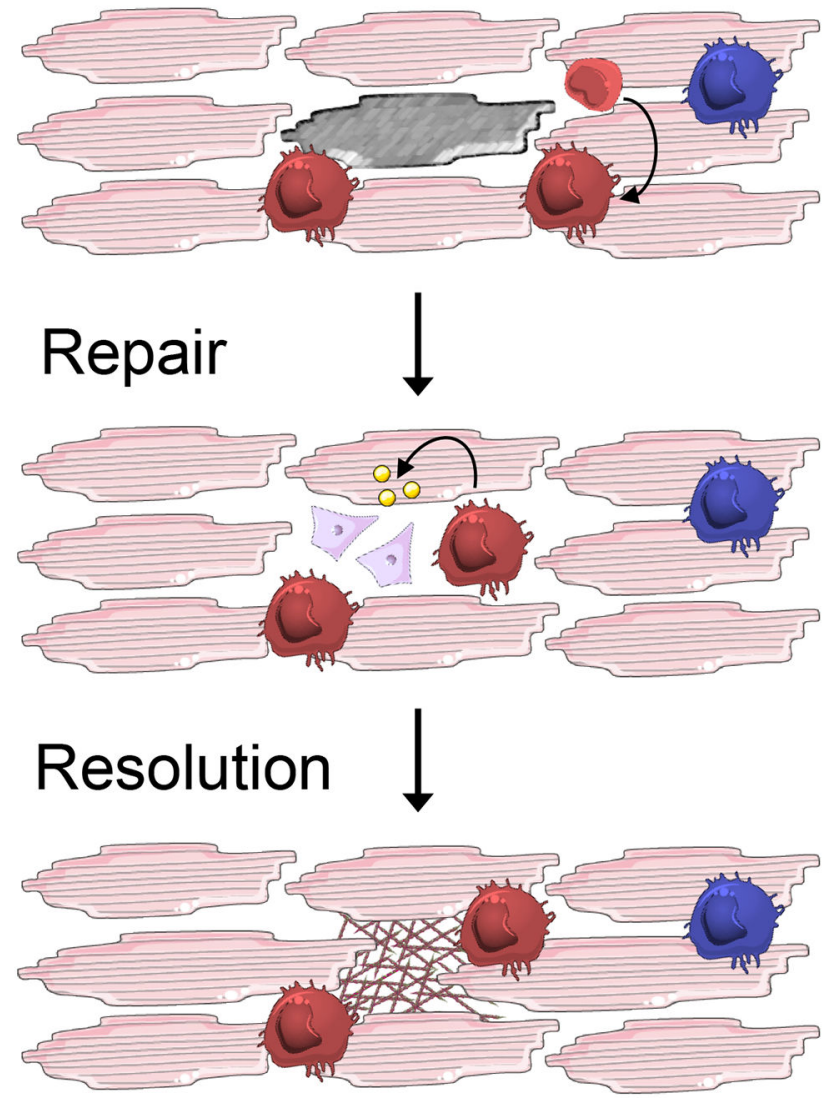

Figure 1. Inflammation Resolution is an active process.

Tissue repair is characterized by 3 phases: inflammation, repair, and resolution.

Inflammation can be initiated both locally or systemically and functions to eliminate the source of injury and remove damaged tissue. In the heart, it is characterized by local cell death, loss of CCR2- resident macrophages, and replacement by recruited CCR2 ${ }^{+}$ monocyte-derived macrophages. Repair is initiated after engulfment and clearance of apoptotic cardiomyocytes, stimulating the release of cytokines, including IL-10 and TGF- $\beta$, which activate fibroblasts to replace and remodel the tissue. Resolution is achieved following elimination of the injurious source and clearance of dead cells and debris. It is characterized by apoptosis and egress of the reparative machinery and restoration of 
homeostasis. In both HFrEF and HFpEF, the failure to resolve inflammation is correlated with pump dysfunction and ultimately, end-stage HF. 

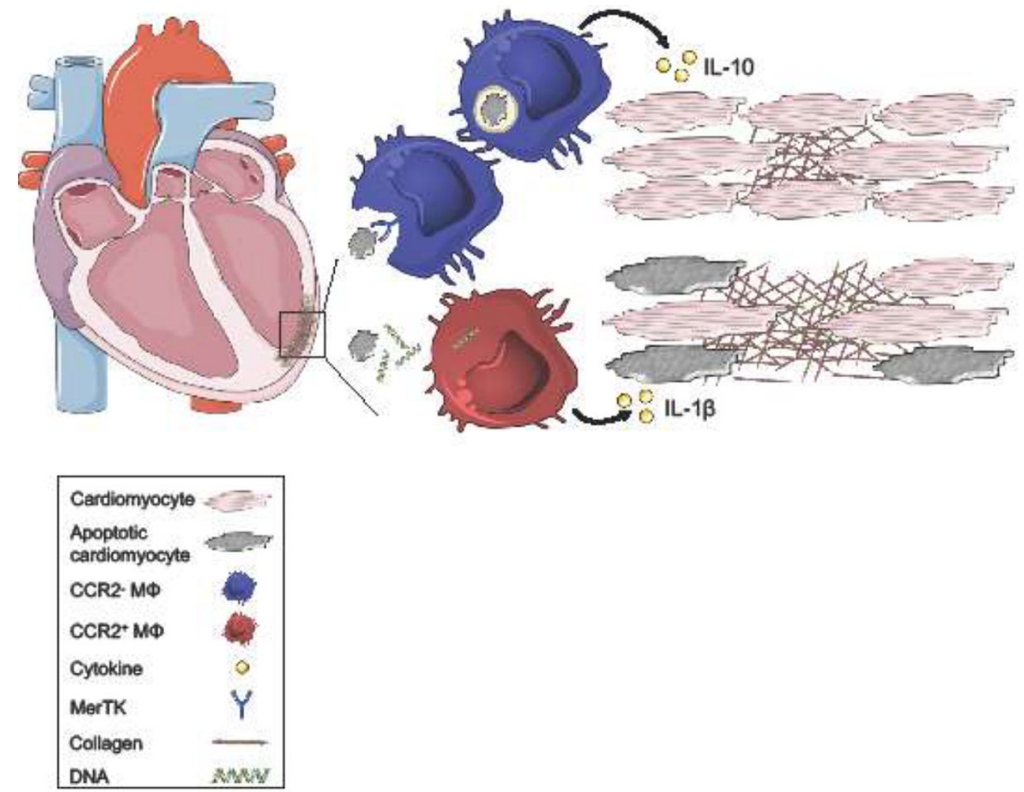

Figure 2. The balance of macrophage inflammatory and reparative responses sets the clinical trajectory in HFrEF.

Following myocardial infarction, cardiomyocyte death primarily occurs through apoptosis and necrosis. Macrophages express surface receptors, including MER proto-oncogene Tyrosine Kinase (MerTK), that recognize and mediate phagocytosis of apoptotic cells. Engulfment and metabolism of dying cells stimulates macrophage production of antiinflammatory cytokines, including IL-10, which preserves neighboring tissue and cardiac function. Dying cardiomyocytes also secrete damage-associated molecular patterns (DAMP), including double-stranded DNA. Macrophage recognition of DAMPs promotes secretion of pro-inflammatory cytokines, including IL-1 $\beta$ leading to collateral tissue damage, adverse ventricular remodeling, and systolic dysfunction. 

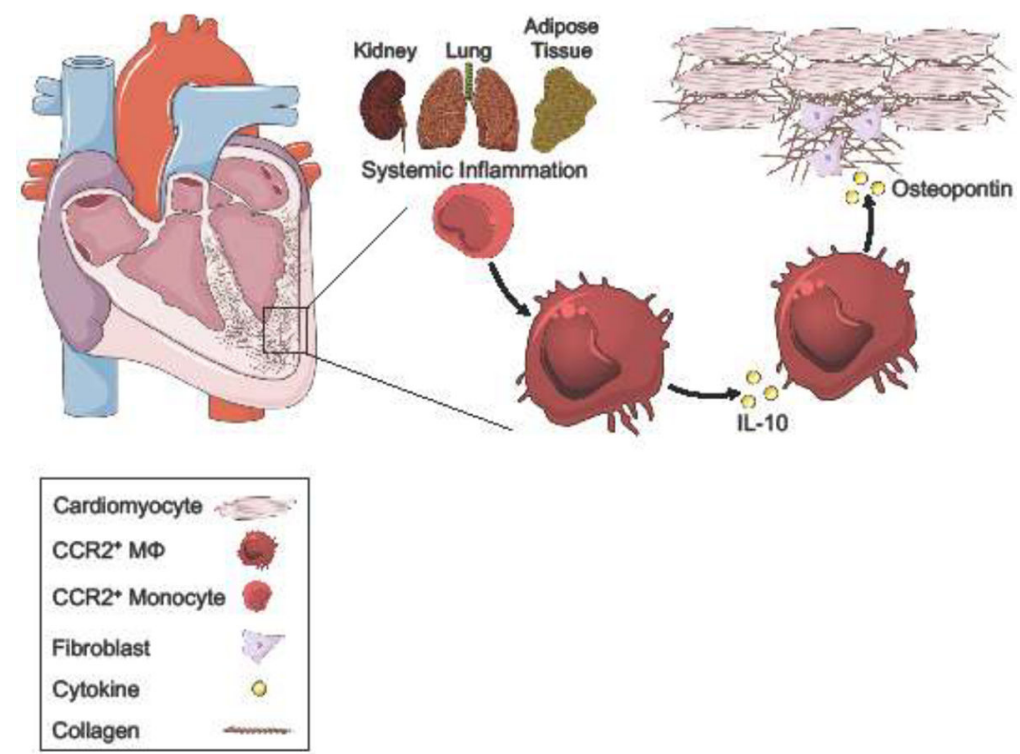

Figure 3. Systemic inflammation perpetuates macrophage repair processes in HFpEF.

The risk of HFpEF increases with age and is associated with an increase in the prevalence of extracardiac comorbidities and risk factors, including chronic kidney disease (kidney), chronic obstructive pulmonary disease (lung), and obesity (adipose tissue). Advanced age and multiple comorbidities in HFpEF patients contribute to a heightened level of systemic inflammation, which promotes myocardial infiltration of $\mathrm{CCR} 2^{+}$monocytes and differentiation into cardiac macrophages. Expression of IL-10 by macrophages leads to autocrine induction of osteopontin, a cytokine associated with cardiac fibrosis. Osteopontin activates cardiac fibroblasts, promoting excess collagen deposition and interstitial fibrosis. These pathologic processes impair cardiomyocyte relaxation and contribute to diastolic dysfunction. 